\title{
Blindness with Superior Vena Cava Obstruction after Cardiac Surgery
}

\author{
H. Algethamy, D. Nicolle, R. Novick, A. Saito, G.B. Young
}

Can. J. Neurol. Sci. 2010; 37: 897-900

Visual loss noted after cardiac surgery occurs in $0.06-0.11 \%$ of cases, due to ischemic damage to either the anterior visual pathway (eyes or optic nerves) or the occipital lobes (cortical blindness). ${ }^{1-6}$ The pupillary light reflex is impaired with ischemic damage to the anterior visual pathway but is spared with cortical blindness. Unilateral or asymmetric bilateral optic nerve involvement produces a relative afferent pupillary defect, but bilaterally symmetrical, severe optic nerve damage results in reduction or loss of the light reflex. While cortical blindness is commonly due to embolic infarction or severe hypotension, the mechanism for optic neuropathy $(\mathrm{ON})$ is less clear.

We have encountered two patients within nine months of each other, in whom ON with blindness was noted shortly after cardiac surgery complicated by superior vena cava (SVC) obstruction.

\section{REPORT OF CASES}

\section{Case 1}

A 58-year-old woman was admitted with congestive heart failure secondary to severe mitral regurgitation. The patient underwent the third redo sternotomy for mitral valve replacement in December, 2006. The patient previously had three other cardiac surgical procedures: an aortic valvotomy and two aortic valve replacements for congenital aortic stenosis. No significant changes in blood pressure, hemoglobin concentration or arterial blood gases were documented perioperatively (transient hypotension intra and postoperatively was treated promptly by fluid boluses and vasopressor agents). Central venous pressure was monitored throughout the surgery and was normal except for one reading of $38 \mathrm{~mm} \mathrm{Hg}$ (markedly elevated) one hour before the conclusion of cardiopulmonary bypass. It was not recorded again. Immediately post-operatively it was recognized that the patient had SVC obstruction, likely secondary to scarring of the superior vena cava-right atrial junction from previous surgeries, manifested by marked bilateral swelling of the head and neck, and confirmed by computed tomography (CT) of the thorax. The SVC obstruction was treated medically because of high risk of surgical intervention in this patient due to multiple surgeries in the past. The patient remained sedated and ventilated for five days until she was stabilized. When the patient was extubated five days after the operation, she complained of complete loss of vision in both eyes. Her neurological examination was normal. Computed tomography and CT angiography of the head and neck, which were done on the fifth post-operative day, were negative except for dilated superior ophthalmic veins (Figure 1). A CT head scan done two years earlier for headaches was normal. The patient was first

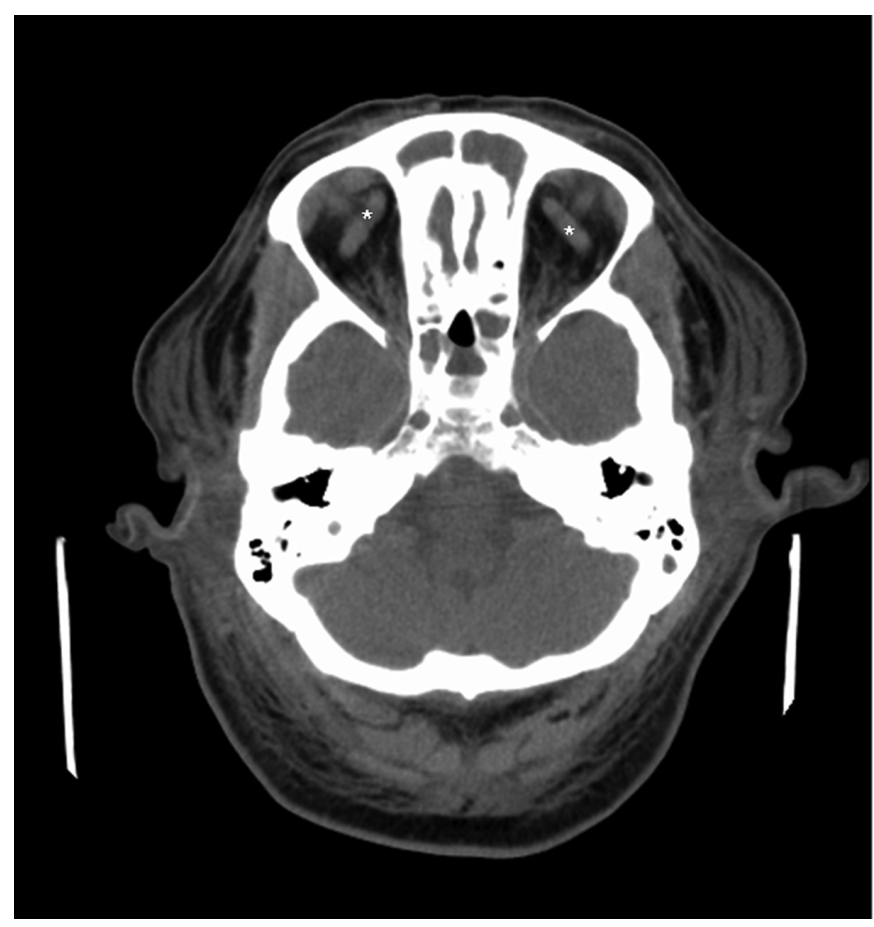

Figure 1: CT scan of the head of Case 1 shows dilated superior ophthalmic veins $(* s)$ when the patient was acutely symptomatic.

seen by an ophthalmologist on the same day of symptomatic visual loss. The exact onset of visual changes was unknown as the patient was sedated. Her ocular examination revealed no light perception in both eyes, bilateral chemosis, and a single right peripheral dot hemorrhage. Both pupils were $8 \mathrm{~mm}$ dilated and did not react to light. Both optic discs and retina were normal. Intraocular pressures were normal. On follow-up three months

From the Departments of Anesthesia (HA), Ophthalmology (DN), Surgery (RN, AS), Clinical Neurological Sciences (GBY), The University of Western Ontario, London, Ontario, Canada.

Received March 31, 2010. Final Revisions Submitted June 10, 2010. Correspondence to: H. Algethamy, Departments of Anesthesia and Neurocritical Care, Room B10-106, University Hospital, 339 Windermere Road, London, Ontario, N6A 5A5, Canada. 
later she had no light perception in both eyes and pupils were unreactive to light. She has remained blind with bilateral optic atrophy.

\section{Case 2}

A 43-year-old woman underwent orthotopic cardiac transplantation for refractory cardiac failure secondary to coronary artery disease in September, 2007. The surgery was complicated by post-operative bleeding. Multiple red blood cell, platelet and fresh frozen plasma transfusions were required, but there was no significant or prolonged hemodynamic instability or change in hematocrit or blood gases. She was extubated 24 hours after surgery, at which time she stated that she could not see. The head and neck were swollen, as in the first patient. The neurological examination was normal and ophthalmological examination revealed absent light perception both eyes. The pupils reacted sluggishly to light. Although there was no chemosis, both fundi showed mild to moderate swelling of optic discs but no associated hemorrhages or cotton wool spots (microinfarcts). Intraocular pressures were not done with this initial examination. A CT scan of the head was negative but that of the chest revealed a stricture of the distal aspect of the SVC. Re-exploration of the mediastinum revealed a large clot compressing the SVC. After clot removal, her hemodynamics stabilized and the remainder of the patient's course, apart from persistent loss of vision, was uneventful. The patient was reassessed two weeks later and her examination showed light perception with left eye (but projection was not accurate) and no light perception with right eye. The left pupil reacted directly to light but the right did not. The optic discs were no longer swollen but both were becoming pale. The intraocular pressures were normal. At the three month visit, she remained blind with right eye but had 20/400 acuity with left eye. Both pupils reacted to light with a right afferent pupillary defect. She had bilateral optic atrophy. She had a constricted field down to $10^{\circ}$ with the left eye. At the seven month post-operative visit, she was able to see hand movements with right eye and 20/25 vision with left eye with a field less than $10^{\circ}$. Both pupils reacted to light with a right afferent pupillary defect and there was bilateral optic atrophy.

\section{Discussion}

Visual loss secondary to ischemic optic neuropathy (ION) is a rare but devastating complication after cardiac surgery. The overall incidence has been reported as $0.06-0.11 \% .3,7$ The etiology of such ION remains poorly understood but the predominant proposed mechanism is critical reduction in blood supply or oxygen carrying capacity to the optic nerve secondary to prolonged hypotension ${ }^{1-6,8,9}$, anemia from intraoperative blood loss ${ }^{1-6,8,9}$, hypoxia ${ }^{1,8}$ and excessive fluid replacement perioperatively. ${ }^{2,5,8,9}$ Based on our recent experience: we wish to propose an additional mechanism in which the venous system is involved. Our cases of optic neuropathy in association with superior vena cava obstruction fit into the syndrome of ischemic optic neuropathy as a form of "perioperative visual loss after nonocular surgeries". 8,10

Perioperative ION is characterized by the sudden development of a painless visual loss and is usually permanent..$^{8,11}$ The visual loss is apparent immediately after awaking from anesthesia after the surgical procedure. ${ }^{2,8,12} \mathrm{We}$

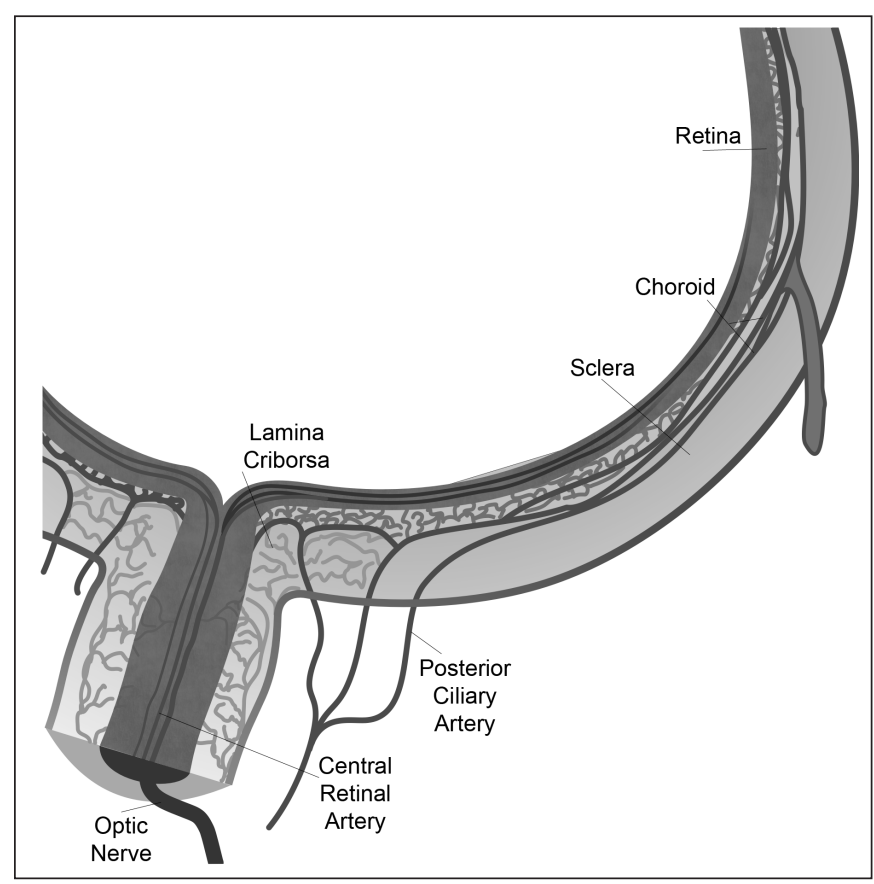

Figure 2: Components of the eye and optic nerve.

agree with Newman that, the delay from the time of surgery is almost certainly a delay in its recognition rather than implying a latent period between the surgery and the optic nerve damage. ${ }^{8}$ In the usual case the patient awakens from surgery with partial or complete bilateral visual loss with poorly reactive or unreactive pupils, and unremarkable neuro-imaging.

Ischemic optic neuropathy is classified into two categories, anterior optic nerve neuropathy (AION) and posterior optic nerve neuropathy (PION). Anterior optic nerve neuropathy results from ischemia of the anterior portion of the optic nerve, and PION is caused by ischemia of the posterior portion of the optic nerve.

Ocular examination in AION typically reveals decreased visual acuity, a relative afferent pupillary defect, visual field loss, and optic disc edema, often with peripapillary hemorrhages, whereas patients with PION present similarly to patients with AION, except that their optic disc is normal acutely, without any disc edema. Occasionally, within a few days of visual loss, a small amount of disc edema may appear as axoplasmic swelling extends forward from the primarily affected retrobulbar optic nerve. ${ }^{13}$ Optic disc pallor ensues four to six weeks later. ${ }^{5,10}$

In this case report, Case 1 had bilateral blindness and there were no funduscopic abnormalities. In Case 2, there was bilateral visual loss and funduscopically revealed disc edema. Therefore, we diagnosed Case 1 as PION and Case 2 as AION or possibly PION, with the disc edema due to an extension of the swelling from the primarily affected retrobulbar optic nerve (as we did not find other changes associated with AION e.g. hemorrhages and cotton wool spots). Both patients had no history of any visual disorders before the surgery. Brain CT did not reveal any 
abnormalities. Magnetic resonance imaging was not done because both patients had permanent pacemakers. Other possible causes of ON had been ruled out through extensive work-up for both patients indicating that the probable cause of ION was related to the surgical procedures.

Knowledge of the vascular anatomy of the optic nerve is helpful in understanding the mechanisms of ischemic optic neuropathies. Blood supply to the optic nerve is provided by two routes: the posterior ciliary artery, which supplies the anterior portion of the nerve and primarily the optic nerve head, whereas the pial vessels, which are supplied by collateral vessels that are independent of the ophthalmic artery, perfuse the posterior part of the optic nerve. ${ }^{14}$ Anterior optic nerve neuropathy results from an infarction in the watershed area between the posterior ciliary artery and the pial vessels. Posterior optic nerve neuropathy may occur because of the tenuous blood supply to the posterior portion of the nerve.

Of the various proposed mechanisms for ION, anemia and hypotension seem unattractive possibilities, as such complications are common in cardiac surgery, yet ION is rare. Also, it seems unlikely that the optic nerves would be the only structures to suffer from low oxygen delivery or hypotension. The most common surgeries associated with ION are spinal surgery in the prone position, cardiac surgery and radical neck dissections (often with ligation of the jugular veins) ${ }^{8}$ In each of these there is the risk of increased venous pressure in the head and neck structures. Bilateral PION with dilated superior ophthalmic veins and initially normal fundi was reported in a patient who had a prolonged operation for spinal stenosis while in the prone position (there was no significant hypotension during the operation). ${ }^{15}$

The cavernous sinus, into which the superior ophthalmic and posterior ciliary veins drain, is more compressible than other intracranial venous sinuses and thus may more readily transmit raised intracranial pressure from superior vena cava obstruction to these vessels. ${ }^{16} \mathrm{We}$ hypothesize that venous hypertension secondary to SVC obstruction embarrasses the arteriolar circulation leading to ischemia of the optic nerve. The optic nerve courses posteriorly from the globe through the optic foramen where a narrowing, the lamina cribrosa, encases the nerve (Figure 2). It is in this area of the scleral canal that compression of the optic nerve can occur in cases of AION. The site for PION may relate to the area that is proximal from the lamina cribrosa or within the optic canal. ${ }^{17}$ Because of crowding of the nerve fibers within the optic canal or a small canal at the lamina cribrosa, edema formation induced by venous congestion increases pressure within the optic nerve leading to a decrease in arteriolar perfusion pressure of the optic nerve, causing further ischemia. The posterior optic nerve may be particularly susceptible to increased venous pressure because of its blood supply. Hayreh proposes that the posterior part of the optic nerve has a varied arterial supply, often from collateral arterioles outside of the optic nerve, i.e., a centripetal pattern. The differential pressure between veins and arterioles determines the perfusion of the nerve. ${ }^{10}$

In our cases, Case 1 had face and neck swelling, bilateral chemosis, a single right peripheral dot hemorrhage and dilated superior ophthalmic veins on brain CT, all compatible with venous hypertension secondary to SVC obstruction. We propose that this led to arterial hypoperfusion of the optic nerve. Case 2 had face and neck swelling on clinical examination and SVC obstruction was confirmed by chest CT. Unlike Case 1, she did not have venous congestion on ocular examination, most likely because the SCV obstruction was recognized and treated early. Blindness in association with SVC obstruction has been reported in a patient with extensive resection of a metastatic germ cell tumor in the right upper mediastinal region. ${ }^{18}$ In that case papilledema was noted shortly after the operation and blindness with secondary optic atrophy was found a month later, after the patient was extubated. We presume that the cause of blindness in the above case report is similar to our cases.

In our cases the brain was spared from venous engorgement and cerebral edema likely because of alternative venous drainage through the vertebral plexus, which allows for effective drainage of the brain's venous blood while the patient is in the supine position. ${ }^{19}$ In addition, there are more compensatory mechanisms for adjusting to raised venous pressure in the cranial compartment than in the optic nerve sheath. Also reductions in blood and cerebrospinal fluid volumes ameliorate rises in intracranial pressure. $^{20}$

We suggest that extra care be taken to avoid superior cava obstruction, including consistent intraoperative monitoring of central venous pressure, and that when such complications are first recognized that they be treated promptly.

Obviously, much further investigation needs to be done in order to fully clarify the risk factors and mechanisms of optic nerve damage in the perioperative setting. A major step would be the development of a valid animal model, particularly for ION in the setting of elevated orbital venous pressures, as likely occurs in complicated cardiac surgery, radical neck dissection and in long prone procedures.

\section{ACKNOWLEDGEMENT}

The authors thank Drs. Mohamed Alzahwahmah and Alex Fraser for helpful advice.

\section{REFERENCES}

1. Torossian A, Schmidt J, Schaffartzik W, et al. Loss of vision after non-ophthalmic surgery: systematic review of the literature on incidence, pathogenesis, treatment and prevention. Anaesthetist. 2006; 55:457-64

2. Buono LM, Froozan R. Perioperative posterior ischemic optic neuropathy. Surg Ophthalmol. 2005; 50:15-26.

3. Kalyani SD, Miller NR, Dong LM, et al. Incidence of and risk factors for perioperative optic neuropathy after cardiac surgery. Ann Thorac Surg. 2004; 78:34-7.

4. Sweeney PJ, Breuer AC. Ischemic optic neuropathy: a complication of cardiopulmonary bypass surgery. Neurology.1982; 32:560-2 .

5. Shapira O, Kimmel W. Anterior ischemic optic neuropathy after open heart operation. Ann Thorac Surg. 1996; 61:660-6.

6. Shaw P, Bates D, Cartlidge N, et al. Neuro-opthalmological complications of coronary artery bybass graft surgery. Acta Neurol Scand. 1987; 76:1-7.

7. Nuttall GA, Garrity JA, Dearani JA, Abel MD, Schroeder DR, Mullany CJ. Risk factors for ischemic optic neuropathy after cardiopulmonary bypass: a matched case/control study. Anesth Analg. 2001; 93:1410-6.

8. Newman NJ. Perioperative visual loss after nonocular surgeries. Am J Ophthalmol. 2008; 145:604-10.

9. Sadda SR, Nee M, Miller NR, Biousse V, Newman NJ, Kouzis A. Clinical spectrum of posterior ischemic optic neuropathy. Am J Ophthalmol. 2001; 132:743-50. 
10. Hayreh SS. Posterior ischaemic optic neuropathy: clinical features, pathogenesis, and management. Eye (Lond). 2004; 18:1188-206.

11. Mathews MK. Nonarteritic anterior ischemic optic neuropathy. Curr Opin Ophthalmol. 2005; 16(6):341-5.

12. Lee LA, Roth S, Posner KL, et al. The American Society of Anesthesiologists postoperative visual loss registry. Anesthesiology. 2006; 105:652 -9.

13. Luneau K, Newman NJ, Biousse V. Ischemic optic neuropathies. Neurologist. 2008; 14:341-54.

14. Hayreh SS. Anterior ischemic optic neuropathy. III. Clinical features and pathogenesis of post-hemorrhagic amaurosis. Ophthalmology. 1987; 94:1488-502.

15. Reddy A, Foroozan R, Edmond JC, et al. Dilated superior ophthalmic veins and posterior optic neuropathy after prolonged spine surgery. J Neuroophthalmol. 2008; 28:327-8.
16. Chen W-T, Fuh J-L, Lirng J-F, et al. Collapsed superior ophthalmic veins in patients with intracranial hypotension. Neurology. 2003; 61:1265-7.

17. Kudo D, Yamamura H, Nishiuchi T, et al. Anterior and posterior ischemic optic neuropathy related to massive fluid resuscitation after blunt trauma. J Trauma. 2010; 68:E67-70.

18. Akkerdijk GP, Van Geel AN. Blindness. A rare and serious complication after extensive mediastinal resection. Ann Thorac Surg. 2008; 85:1426-7.

19. Epstein HM, Linde HW, Crompton AR, et al. The vertebral venous plexus as a major cerebral venous outflow tract. Anesthesiology. 1970; 32:332-7.

20. Steiner LA, Andrews PJ. Monitoring the injured brain: ICP and CBF. Br J Anaesth. 2006; 97(1):26-38. 